\title{
Cancer-derived exosomes induce immunosuppressive macrophage polarization in bladder cancer
}

\section{Ziming Jiang \\ Yiming Zhang \\ Yu Zhang \\ Zhankui Jia \\ Zhengguo Zhang \\ Jinjian Yang}

\section{Video Byte}

Keywords: exosomes, bladder cancer, tumor-associated macrophages, TAMs, tumor microenvironment, TME, progression, macrophage, cancer, macrophage polarization, immunosuppression, MB49, xenograft, in vitro, PTEN, AKT/STAT3/6, M2 phenotype, microarray, reprogramming, Cell Communication and Signaling

Posted Date: October 13th, 2021

DOI: https://doi.org/10.21203/rs.3.rs-966299/v1

License: (c) (1) This work is licensed under a Creative Commons Attribution 4.0 International License. Read Full License 


\section{Abstract}

The immunosuppressive tumor microenvironment (TME) plays essential roles in cancer development and progression. Exosomes mediate crosstalk between tumor cells and other stromal or immune cells in the TME, but how tumor-derived exosomes promote the progression of bladder cancer, one of the most common types of cancer, remains unclear. To find out, researchers recently examined the effects of exosomes extracted from the conditioned medium (CM) of MB49 bladder cancer cells. The researchers found that the cancer-derived exosomes were ingested by mouse macrophages both in vitro and in vivo and that they induced macrophage polarization toward the immunosuppressive M2 phenotype. Exosomesecreting MB49 cells induced tumor growth in mice, but the exosome inhibitor GW4869 reduced tumor growth, macrophage M2 polarization and immunosuppression, confirming the pro-tumor effects of the cancer-derived exosomes. Microarray and western blot analyses indicated that the exosome-induced macrophage polarization was mediated by PTEN downregulation and AKT/STAT3/ 6 activation and that the exosomal miRNAs miR-1231-5p and miR-92b-3p were involved. Although additional mechanistic investigation is needed, the results suggest that bladder cancer-derived exosomes help establish an immunosuppressive TME and provide a potential therapeutic target for bladder cancer treatment. 